\title{
Proportional Nonuniform Multi-Gabor Expansions
}

\author{
Shidong Li \\ College of Science and Engineering, San Francisco State University, San Francisco, CA 94132, USA \\ Email: shidong@sfsu.edu \\ Received 18 December 2003; Revised 10 May 2004; Recommended for Publication by Helmut Boelcskei
}

\begin{abstract}
A nonuniform multi-Gabor expansion (MGE) scheme is studied under proportional time and frequency (TF) shifts among different window indices $m$. In particular, TF parameters for each $m$ are different, but proportional and relevant to windows' TF patterns. The generation of synthesis waveforms for nonuniform MGE is generally difficult. We show constructively that there is a set of basic synthesis MGE waveforms at each window index under proportional parameter settings. Nonuniform MGE adapts to signal frequency dynamics effectively, and eliminates unnecessary overlapping redundancies of a uniform MGE. Examples of the evaluation of synthesis waveforms are provided. Efficiency comparison of TF analysis using nonuniform and uniform MGEs is also discussed.
\end{abstract}

Keywords and phrases: nonuniform time-frequency shifts, multi-Gabor representations, time-frequency analysis, frames, dual frames.

\section{INTRODUCTION}

Typical Gabor expansions use one fixed window and its translates and complex modulates as basic elements in an attempt to analyze the time-frequency (TF) information of a signal. Studies on Gabor expansions are intense; for example, see $[1,2,3,4,5,6,7,8,9,10,11,12,13,14,15,16]$. There is also a book on Gabor analysis and algorithm by Feichtinger and Strohmer [17]. However, frequency-varying features of a signal or the multifrequency components in a signal require windows of different size (variance) for a refined TF resolution. Multi-Gabor expansions (MGEs) were developed specifically to meet such requirements; for example, see $[11,18]$, (cf. $[19,20]$ ). MGEs are TF expansions using a set of multiple windows and their translates and modulates in a frame (overcomplete "basis") system. The set of windows are custom-tuned. Typically, they range from a narrower window to a wider window to meet the requirements of TF representations of signals of timevarying frequency components. However, standard uniform MGEs apply the same TF shifts among all analysis windows. Such uniform MGEs do not take into consideration the distinct TF patterns of different windows, which gives rise to unnecessary redundancy. Nonuniform MGE schemes adapted to each window's TF characteristics are more natural.

Let $0 \leq m \leq M-1$ be the number of windows in an MGE system. Let $j$ and $k$ be the modulation and translation parameters, and let $\mathscr{H}_{L}$ be an $L$-dimensional signal space. Then a discrete nonuniform multi-Gabor representation (of nonuni- form TF shifts) is defined by, for all $s \in \mathscr{H}_{L}$,

$$
s=\sum_{m=0}^{M-1} \sum_{j=0}^{N_{m}-1} \sum_{k=0}^{K_{m}-1}\langle s, \gamma(m, j, k)\rangle g_{j / N_{m}}^{(m)}, k T_{m},
$$

where, for the window index $m, N_{m}$ is the number of frequency bins $\left(1 / N_{m}\right.$ is the frequency shift), $T_{m}$ is the timetranslation parameter, $K_{m} \equiv L / T_{m}, g^{(m)}$ is the $m$ th window, and $\{\gamma(m, j, k): m, j, k\}$ is a set of synthesis sequences to be constructed. We have also used the conventional notation $\left\{g_{j / N_{m}, k T_{m}}^{(m)}\right\}$ for the given multi-Gabor family, namely,

$$
\left\{g_{j / N_{m}, k T_{m}}^{(m)} \equiv g^{(m)}\left(\cdot-k T_{m}\right) e^{\left(2 \pi i / N_{m}\right) j(\cdot)}\right\}_{m, j, k},
$$

where $(\cdot)$ is the (time) variable of the function. In $\mathcal{H}_{L},(\cdot)$ is the index $n$, where $n=0,1, \ldots, L-1$.

MGEs are frame representations. A frame in a Hilbert space $\mathscr{H}$ is a basis-like sequence $\left\{x_{n}\right\}$ for which there are constants $0<A \leq B<\infty$ such that

$$
A\|x\|^{2} \leq \sum_{n}\left|\left\langle x, x_{n}\right\rangle\right|^{2} \leq B\|x\|^{2} \quad \forall x \in \mathscr{H} .
$$

If $\left\{x_{n}\right\}$ is a frame, the standard dual-frame representation stands for,

$$
\forall x \in \mathscr{H}, \quad x=\sum_{n}\left\langle x, S^{-1} x_{n}\right\rangle x_{n}=\sum_{n}\left\langle x, x_{n}\right\rangle S^{-1} x_{n},
$$


where $S^{-1}$ is the inverse of the standard frame operator $S$ defined by,

$$
\forall x \in \mathcal{H}, \quad S x=\sum_{n}\left\langle x, x_{n}\right\rangle x_{n} .
$$

The sequence $\left\{S^{-1} x_{n}\right\}$ is the standard dual frame to the frame $\left\{x_{n}\right\}$. In the case of nonuniform MGEs, $\left\{g_{j / N_{m}, k T_{m}}^{(m)}\right\}$ is to form a frame in $\mathscr{H}_{L}$, and $\{\gamma(m, j, k): m, j, k\}$ would be a dual frame to $\left\{g_{j / N_{m}, k T_{m}}^{(m)}\right\}$. A dual frame needs not be the standard dual. One can have infinitely many constructible dual frames for a frame that is redundant [21]. We will only be focusing on standard duals here. Gabor duals in a parametric form can be found in [12]. The same dual formula can be transformed to nonuniform multi-Gabor scenarios discussed here.

\section{Gabor expansions from a filtering point of view}

A typical uni-Gabor expansion (with one window) is equivalent to shifted bandpass filtering in signal processing. The bandwidth of the filter is determined by the window size. Wider window in time has typically narrower frequency bandwidth and vice versa. Evidently, a fixed bandwidth system is insufficient to analyze signals of dynamic frequencies-a phenomenon known as poor resolution. This was the very reason why the multi-Gabor representations, for example, see $[11,18]$, was introduced. With multi-Gabor systems, there are now multiple bandpass filters of varying bandwidth. They can be designed to adapt to signal frequency dynamics, and thereby provide flexible and adaptive TF analysis of a signal.

\section{Reasons for using nonuniform TF shifts}

While MGE marks one step forward in a refined TF analysis of Gabor expansions, uniform MGE schemes do not reflect the nature of TF patterns of different windows, causing excessive and unnecessary TF-tiling overlaps.

For instance, a wider window in time has narrower effective frequency bandwidth and vice versa. Hence, in a more natural MGE scheme, a wider window in time should be assigned with larger time translates and smaller frequency shifts (i.e., larger $N_{m}$ ). Likewise, a narrower window should be given smaller time but larger frequency shifting parameters.

MGEs of nonuniform time translates but fixed frequency shifts among windows have been analyzed and constructed in detail in [18].

When both TF shifts are varying among window indices, the theory and construction of nonuniform MGEs are generally difficult. In this paper, we report the study on the construction of a nonuniform MGE with proportional TF shifts, namely, the time translates and frequency shifts are proportional among window indices and relevant to windows' TF patterns, following the basic TF tiling principle described earlier.

We provide an analysis and construction of the structure of the dual multi-Gabor frames of such proportional nonuniform MGEs. We show that the standard dual multiGabor frame is formed by translations and modulations of a set of basic windows at each window index $m$. This result in turn allows us to derive a fast algorithm for the evaluation of these basic dual windows. Numerical examples of the construction of such MGEs as well as the efficiency discussion of nonuniform MGEs in TF analysis are also presented.

\section{CONSTRUCTING NONUNIFORM MGES}

To construct a nonuniform MGE system, one has to make sure that a set of multi-Gabor waveforms form a frame.

Intuitively, the requirement is to have combined window waveforms covering the entire TF plane. We show in the following some direct conditions for the construction of nonuniform MGEs. This will provide users with an intuitive guideline. The proof of the following result can be found in Appendix A.1.

Theorem 1. Let $\left\{g^{(m)}\right\}_{0}^{M-1} \in \mathscr{H}_{L}$ be a set of $M$ window functions. For each $m$, let $T_{m}$ and $N_{m}$ be the translation and modulation parameters, respectively, satisfying $K_{m} T_{m}=L$ and $P_{m} N_{m}=L$ with integers $K_{m}$ and $P_{m}$. Assume that

(1) $A \leq \sum_{m=0}^{M-1} N_{m} \sum_{k=0}^{K_{m}-1}\left|g^{(m)}\left(n-k T_{m}\right)\right|^{2} \leq B$ for some $0<A \leq B<\infty$, and for all $n$, (2) $\sum_{\text {that }}^{M-1} N_{m} \sum_{l_{m}=1}^{P_{m}-1} a^{(m)}\left(l_{m} N_{m}\right)$ is sufficiently small such

$$
A-\sum_{m=0}^{M-1} N_{m} \sum_{l=1}^{P_{m}-1} a^{(m)}\left(l N_{m}\right) \geq A_{0}>0
$$

where

$$
a^{(m)}\left(l N_{m}\right) \equiv \max _{0 \leq q \leq L-1}\left|\sum_{k=0}^{K_{m}-1} g^{(m)}\left(q-k T_{m}\right) \overline{g^{(m)}\left(q+l N_{m}-k T_{m}\right)}\right| .
$$

Then, $\left\{g_{j k}^{(m)}\right\}_{m, j, k}\left(0 \leq m \leq M-1,0 \leq j \leq P_{m}-1\right.$, and $0 \leq$ $\left.k \leq K_{m}-1\right)$ forms a multi-Gabor frame of $\mathcal{H}_{L}$.

Remark 1. (a) Notice that the indices $j, k$, and $l$ should all have carried a subindex $m$ as they all have different ranges for different $m$. Nevertheless, the subindex $m$ is omitted for the simplicity of the presentation.

(b) If the combined translates of all windows cover all the signal span (condition (1)), and if $N_{m}$ is large enough (so that the relative shifts in the cross-correlation $a^{(m)}\left(l N_{m}\right)$ is amply extended) to sufficiently lower the value of $a^{(m)}\left(l N_{m}\right)$ (condition (2)), then $\left\{g_{j k}^{(m)}\right\}$ forms a nonuniform multi-Gabor frame.

It is also possible to improve the conditions in Theorem 1 following an observation by Casazza and Christensen [22].

Theorem 2. Let $\left\{g^{(m)}\right\}_{0}^{M-1} \in \mathscr{H}_{L}$ be a set of $M$ window functions. For each $m$, let $T_{m}$ and $N_{m}$ be given in Theorem 1 . 
Assume that

(1)

$$
\begin{aligned}
& \inf _{n} \sum_{m=0}^{M-1} N_{m}\left(\sum_{k=0}^{K_{m}-1}\left|g^{(m)}\left(n-k T_{m}\right)\right|^{2}\right. \\
& -\sum_{l=1}^{P_{m}-1} \mid \sum_{k=0}^{K_{m}-1} g^{(m)}\left(n-k T_{m}\right) \\
& \left.\times \overline{g^{(m)}\left(n+l N_{m}-k T_{m}\right)} \mid\right)=a>0,
\end{aligned}
$$

(2)

$$
\begin{aligned}
& \sup _{n} \sum_{m=0}^{M-1} N_{m} \sum_{l=0}^{P_{m}-1}\left|\sum_{k=0}^{K_{m}-1} g^{(m)}\left(n-k T_{m}\right) \overline{g^{(m)}\left(n+l N_{m}-k T_{m}\right)}\right| \\
& \quad=b<\infty .
\end{aligned}
$$

Then, $\left\{g_{j k}^{(m)}\right\}_{m, j, k}\left(0 \leq m \leq M-1,0 \leq j \leq P_{m}-1\right.$, and $0 \leq k \leq K_{m}-1$ ) forms a multi-Gabor frame of $\mathscr{H}_{L}$.

A sketch of the proof can be found in Appendix A.2. Note that condition (2) of Theorem 2 may be removed in finitedimensional space since it always holds. Nevertherless, since the ratio of the frame bounds determines the numerical stability of the frame system, condition (2) can be used towards that end.

The improvement of conditions in Theorem 2 lies in the fact that pointwise comparison is done between the sampled autocorrelation $\sum_{k=0}^{K_{m}-1}\left|g^{(m)}\left(n-k T_{m}\right)\right|^{2}$ and the sampled cross-correlation (shifted correlation) $\sum_{l=0}^{P_{m}-1}\left|\sum_{k=0}^{K_{m}-1} g^{(m)}\left(n-k T_{m}\right) \overline{g^{(m)}\left(n+l N_{m}-k T_{m}\right)}\right|$, while in Theorem 1, the infimum of the autocorrelation must be greater than the supreme of the cross-correlation. When the redundancy of a nonuniform MGE system is close to the minimum, the conditions in Theorem 2 will become substantially superior.

The remaining fundamental task is to determine dual multi-Gabor waveforms $\left\{\gamma^{(m, j, k)}\right\}$ for a given multi-Gabor (frame) sequence $\left\{g_{j k}^{(m)}\right\}$.

\section{NONUNIFORM MGES WITH FIXED PROPORTIONS OF TF SHIFTS}

Assume that the window "width" increases as index $m$ increases (from 0 to $M-1$ ), and hence assume that $T_{m}$ increases. Since a wider window corresponds to narrower frequency bandwidth, a smaller frequency shift is desired, which in turn corresponds to a larger number of frequency bin $N_{m}$ as see in (2). Therefore, both $T_{m}$ and $N_{m}$ are assumed increasing as $m$ goes up.

Let $T=T_{M-1}$, the largest time translate, and let $N=N_{0}$, the smallest frequency bin (which corresponds to the largest frequency shift). Then, for integers $\alpha$ and $\beta$, we assume that the following proportional relationship hold:

$$
\begin{gathered}
N_{m} \equiv \alpha N_{m-1}=\alpha^{m} N, \quad \alpha \geq 1, \\
T_{m} \equiv \beta T_{m-1}=\beta^{m-M+1} T, \quad \beta \geq 1 .
\end{gathered}
$$

We show that the standard dual sequences $\{\gamma(m, j, k)$ : $m, j, k\}$ are formed by translations and modulations of several basic window waveforms together with multiplications of relevant constants. More precisely, we have the following result. Notation $e_{p / N} \tau_{q T} x \equiv x(\cdot-q T) e^{2 \pi i p(\cdot) / N}$ is used from time to time for convenience.

Theorem 3. Let $\left\{g_{j k}^{(m)}\right\}_{m, j, k}$ be a given multi-Gabor frame for $\mathcal{H}_{L}$. Assume that $T_{m}$ and $N_{m}$ for the index $m$ are defined by (10), respectively. Let $j=u \alpha^{m}+p$ and $k=v \beta^{M-m-1}+q$, where $0 \leq u \leq N-1,0 \leq p \leq \alpha^{m}-1,0 \leq v \leq L / T-1$, and $0 \leq q \leq \beta^{(M-m-1)}-1$, and so that $0 \leq j \leq N_{m}-1$ and $0 \leq k \leq L / T_{m}-1$. Then, at the window index $m$,

(1) there are $\alpha^{m} \beta^{(M-m-1)}$ basic dual multi-Gabor windows $\left\{\tilde{\gamma}^{(m, p, q)}: 0 \leq p \leq \alpha^{m}-1,0 \leq q \leq \beta^{(M-m-1)}-1\right\} ;$

(2) these basic $\left\{\tilde{\gamma}^{(m, p, q)}\right\}$ are given by

$$
\begin{gathered}
\tilde{\gamma}^{(m, p, q)}=S^{-1}\left[g_{p / N_{m}, q T_{m}}^{(m)}\right], \\
\forall 0 \leq p \leq \alpha^{m}-1, \\
\forall 0 \leq q \leq \beta^{(M-m-1)}-1,
\end{gathered}
$$

where $S$ is the frame operator defined by the multi-Gabor frame $\left\{g_{j k}^{(m)}\right\}_{m, j, k}$ :

$$
\forall x \in \mathscr{H}_{L}, \quad S x=\sum_{m} \sum_{j k}\left\langle x, g_{j k}^{(m)}\right\rangle g_{j k}^{(m)} .
$$

The standard dual multi-Gabor sequence $\left\{\gamma_{u / N, v T}^{(m, p, q)}: p, q, u, v\right\}$ at index $m$ is formed by

$$
\gamma_{u / N, v T}^{(m, p, q)} \equiv e_{u / N} \tau_{v T}\left[e^{2 \pi i p v T / N_{m}} \tilde{\gamma}^{(m, p, q)}\right]
$$

for all $0 \leq u \leq N-1,0 \leq v \leq L / T-1$, and for all $p$ and $q$ specified in (11). Here $T=T_{M-1}$ and $N=N_{0}$ are the largest time-shifting step and the smallest frequency bins, respectively.

A proof of the result can be found in Appendix B.

Remark 2. (a) Theorem 3 states that the construction of a nonuniform MGE with proportional TF shifts is reduced to the evaluation of a set of basic dual window waveforms $\gamma^{(m, p, q)}$ at each index $m$ by (11). This result greatly facilitates the evaluation of proportional nonuniform multiGabor dual frames. It also allows us to derive a fast algorithm for the construction of the dual multi-Gabor frames.

(b) The standard dual multi-Gabor sequences $\left\{\gamma_{u / N, v T}^{(m, p, q)}\right\}$ are formed by translations of the basic windows $\tilde{\gamma}^{(m, p, q)}$ by the largest step size $T$ and multiplied by a constant $e^{2 \pi i p v T / N_{m}}$ (for each parameter $p$ and each translate $v$ ) and then modulated by the smallest frequency bins $N$. Notice that the constant $e^{2 \pi i p v T / N_{m}}$ is mostly 1 if $p v T / N_{m}$ is an integer. 
In particular, if $N_{m}$ divides $T$ for all $m$ (which is practically so since $T$ is the largest translation parameter among all), the constant is then always 1. Examples will be presented later.

For instance, let $M=2$ and $\alpha=\beta=2$. We have a nonuniform MGE of 2-window system. Then for the window index $m=0$, there are 2 basic dual windows for $p=0$ and $q=0,1$. For $m=1$, there are 2 basic dual windows as well for $p=0,1$ and $q=0$. Moreover, the 2 basic dual windows for $m=0$ are both real-valued windows $(p=0)$, while the second basic dual windows for $m=1$ are generally complex valued since $p=1$. See (11).

\section{NONUNIFORM MGES WITH VARYING PROPORTIONS OF TF SHIFTS}

While MGEs of fixed proportional TF shifts (presented in the last section) are natural enough for a majority of applications, it is conceivable that some applications may demand a nonuniform MGE scheme, where the TF shifts do not follow a fixed proportion. Under an assumption that each of the translation and modulation parameters $T_{m}$ and $N_{m}$ satisfies an integer-multiple relationship with respect to the reference parameter set $(T, N)$, it is possible to analyze the dual structure similarly.

Again, let $T=T_{M-1}$, the largest time translate, and let $N=N_{0}$, the smallest frequency bin parameter. For integers $\alpha_{m}$ and $\beta_{m}$ for all $m$, we now assume that

$$
\begin{array}{cc}
N_{m} \equiv \alpha_{m} N, & \alpha_{m} \geq 1, \\
T \equiv \beta_{m} T_{m}, & \beta_{m} \geq 1 .
\end{array}
$$

It turns out that a similar dual structure result holds.

Theorem 4. Let $\left\{g_{j k}^{(m)}\right\}_{m, j, k}$ be a given multi-Gabor frame for $\mathscr{H}_{L}$. Assume that $T_{m}$ and $N_{m}$ for the index $m$ satisfy (14), respectively. Let $j=u \alpha_{m}+p$ and $k=v \beta_{m}+q$, where $0 \leq u \leq$ $N-1,0 \leq p \leq \alpha_{m}-1,0 \leq v \leq L / T-1$, and $0 \leq q \leq \beta_{m}-1$, and so that $0 \leq j \leq N_{m}-1$ and $0 \leq k \leq L / T_{m}-1$. Then, at the window index $m$,

(1) there are $\alpha_{m} \beta_{m}$ basic dual multi-Gabor windows $\left\{\tilde{\gamma}^{(m, p, q)}: 0 \leq p \leq \alpha_{m}-1,0 \leq q \leq \beta_{m}-1\right\} ;$

(2) these basic $\left\{\tilde{\gamma}^{(m, p, q)}\right\}$ are given by

$$
\begin{aligned}
\tilde{\gamma}^{(m, p, q)} & =S^{-1}\left[g_{p / N_{m}, q T_{m}}^{(m)}\right] \\
\forall 0 & \leq p \leq \alpha_{m}-1, \\
\forall 0 & \leq q \leq \beta_{m}-1,
\end{aligned}
$$

where $S$ is the frame operator defined by the multi-Gabor frame $\left\{g_{j k}^{(m)}\right\}_{m, j, k}$ as in (12).

The standard dual multi-Gabor sequences $\left\{\gamma_{u / N, v T}^{(m, p, q)}: p, q, u, v\right\}$ at index $m$ are formed by (13) for all $0 \leq u \leq N-1,0 \leq v \leq$ $L / T-1$, and for all $p$ and $q$ specified in (15). Here $T=T_{M-1}$ and $N=N_{0}$ are the largest time-shifting step and the smallest frequency bins, respectively.

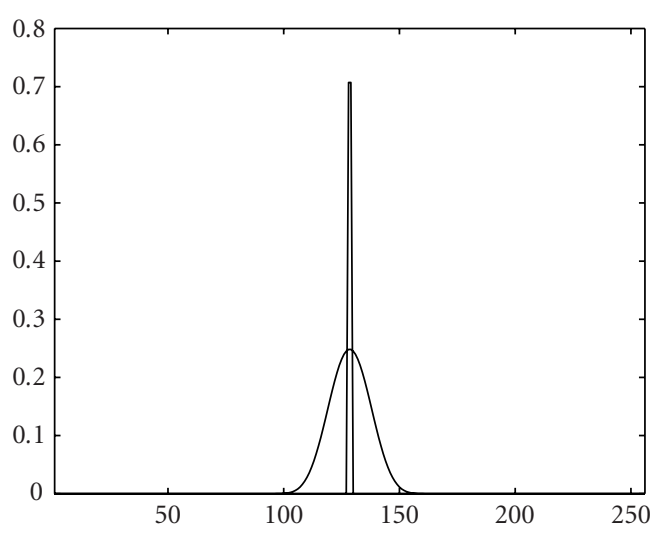

FIgURE 1: A 2-window system.

The proof of this result mimics that of Theorem 3 which can be found in Appendix B.

\section{THE EVALUATION OF THE DUAL WAVEFORMS}

The evaluation of the dual multi-Gabor waveforms takes an algorithmic structure similar to that of uniform frequency shifting. In fact, the frame operator matrix is still a sparse and "banded" matrix corresponding to the smallest frequency bin $N$. The computation of each synthesis waveform at each index $m$ is broken into a set of $N_{m} \times$ $N_{m}$ systems of linear equations. We refer to [18, Algorithm 4] for a similar implementation algorithm that breaks the system into a set of smaller systems of equations. Readers that are interested in the implementation of the construction algorithm may contact the author for the Matlab code.

We present an example of 2-window multi-Gabor systems and their duals with (proportional) nonuniform TF shifts. Figure 1 is the given 2 -window system that generates a multi-Gabor frame. In all figures, the horizontal axis is the time index, and the vertical axis is the magnitude of the window waveform.

Figures 2 and 3 are the sets of dual multi-Gabor windows at two window indices. When pictures are shown in two columns, the left column (a) represents the real parts of the basic dual window functions, while the right column (b) represents the imaginary parts. The parameters in this example are $T=16$ (the largest time-translation parameter), $N=16$ (the smallest frequency-shift parameter); and $\alpha_{0}=1, \alpha_{1}=8$, $\beta_{0}=8$, and $\beta_{1}=1$.

Remark 3. It seems that at $m=0$, the $\beta_{0}$ ( since $\alpha_{0}=1$ ) basic synthesis windows are simply translates of each other. A further notice that can be made is that these basic window functions do have a shifting property, but not exactly the shift to each other in general. This observation applies to both scenarios of fixed as well as varying proportional parameters as in Sections 3 and 4, respectively. 

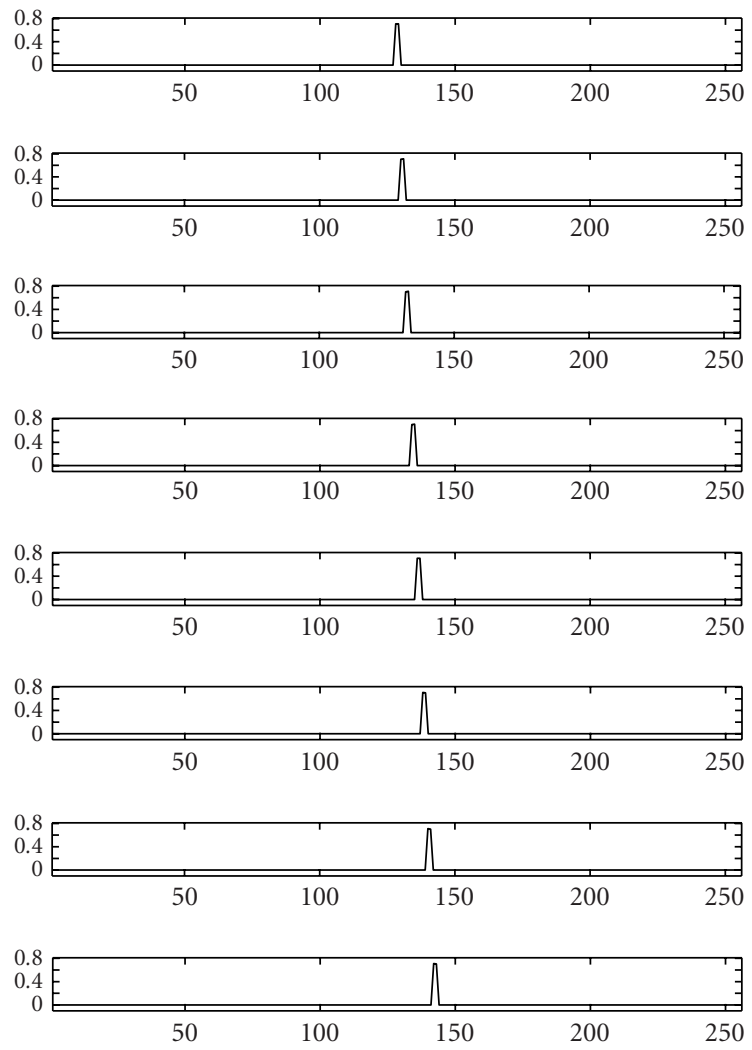

Figure 2: The 8 basic dual multi-Gabor windows at scale $m=0$ : $T_{0}=2, N_{0}=16$.

Corollary 1. Let $\left\{g_{j k}^{(m)}\right\}_{m, j, k}$ be a given multi-Gabor frame for $\mathcal{H}_{L}$ whose parameters $T_{m}$ and $N_{m}$ are given as in Theorem 4. Let $S_{q T_{0}}$ be the frame operator associated with the multi-Gabor frame sequence $\left\{g_{j k}^{(m)}\left(\cdot+q T_{0}\right)\right\}_{m, j, k}$, that is,

$\forall x \in \mathscr{H}_{L}, \quad S_{q T_{0}} x=\sum_{m} \sum_{j k}\left\langle x, g_{j k}^{(m)}\left(\cdot+q T_{0}\right)\right\rangle g_{j k}^{(m)}\left(\cdot+q T_{0}\right)$.

Then the $\beta_{0}$ basic waveforms $\left\{\tilde{\gamma}^{(0,0, q)}\right\}_{q=0}^{\beta_{0}-1}$ at $m=0$ are given by

$$
\tilde{\gamma}^{(0,0, q)}=S^{-1}\left(\tau_{q T_{0}} g^{(0)}\right)=\tau_{q T_{0}}\left(S_{q T_{0}}^{-1} g^{(0)}\right), \quad 0 \leq q \leq \beta_{0}-1 .
$$

A sketch of the proof can be found in Appendix C. Note that, except for index $m=0$, the translation by $T_{m}$ of the window $g^{(m)}\left(\cdot+q T_{0}\right)$ for all $m \neq 0$ no longer covers the signal span in exactly the same way as the $T_{m}$-translates of $g^{(m)}$. Consequently, $S \neq S_{q T_{0}}$ in general. In fact, for each $q$, $S_{q T_{0}}$ is generally different. Nevertheless, since the translates of $g^{(m)}\left(\cdot+q T_{0}\right)$ still cover the signal span, $\left\{g_{j k}^{(m)}\left(\cdot+q T_{0}\right)\right\}_{m, j, k}$ remains a multi-Gabor frame. Because of the redundancy, the difference among $S_{q T_{0}}$ for different $q$ is generally unnoticeable.

\section{A LOOK AT THE EFFICIENCY OF NONUNIFORM MGE}

We consider the efficiency of the proportional nonuniform MGEs by calculating the number of coefficients and by comparing with that of the uniform MGEs, under the assumption that the corresponding TF resolution for each corresponding window stays equivalent.

Take a 2-window system for instance. Assume that one narrower window $(m=0)$ has a support of 8 units, and the wider window $(m=1)$ has 64 units. This system is exactly the same as illustrated in Figures 1, 2, and 3.

The parameter selection follows the principle that the TF resolution is equivalent in corresponding window indices between the proportional nonuniform MGE and the uniform MGE. Specifically, at $m=0$, since the TF pattern is fundamentally stretched in the frequency direction and very narrow in time, the time resolution is the fine one. The uniform MGE parameter $T$ is necessarily small and is set at $T=2$. Meantime, at $m=1$, the TF pattern is stretched in the time direction and very narrow in frequency. The frequency parameter $N$ is set at $N=$ 128 to attain a certain frequency resolution in the uniform MGE.

In the corresponding nonuniform MGE, $T_{0}$ is set at $T_{0}=$ $T=2$. Hence the fine time resolution emphasized at $m=0$ is kept the same between the uniform and nonuniform MGE systems, and $N_{0}$ is set at $N_{0}=16 \ll N=128$. At $m=1$, the fine frequency resolution is to be preserved. Hence, $N_{1}$ is set as $N_{1}=N=128$, while $T_{1}$ is enlarged substantially: $T_{1}=16 \gg T=2$.

With the equivalent TF resolutions, the efficiency improvement of the representation using the nonuniform TF shifts is clearly substantial. The number of coefficients of the proportional nonuniform MGE is

$$
\frac{L}{T_{0}} \times N_{0}+\frac{L}{T_{1}} \times N_{1}=\frac{L}{2} \times 16+\frac{L}{16} \times 128=16 L,
$$

where $L$ is the length of the signal. Meantime, the number of coefficients of the uniform MGE is

$$
2 \times \frac{L}{T} \times N=2 \times \frac{L}{2} \times 128=128 L .
$$

The ratio of the coefficients (nonuniform versus uniform) is merely $12.5 \%$. This is expected to substantially reduce the postprocessing complexity.

\section{CONCLUSION}

We present the results of nonuniform multi-Gabor representations of proportional TF shifts among window indices. Theorems 3 and 4 make possible efficient implementations of the construction of nonuniform multi-Gabor dual-frame systems. It is shown that the standard dual system can be generated by a set of basic waveforms as well. 

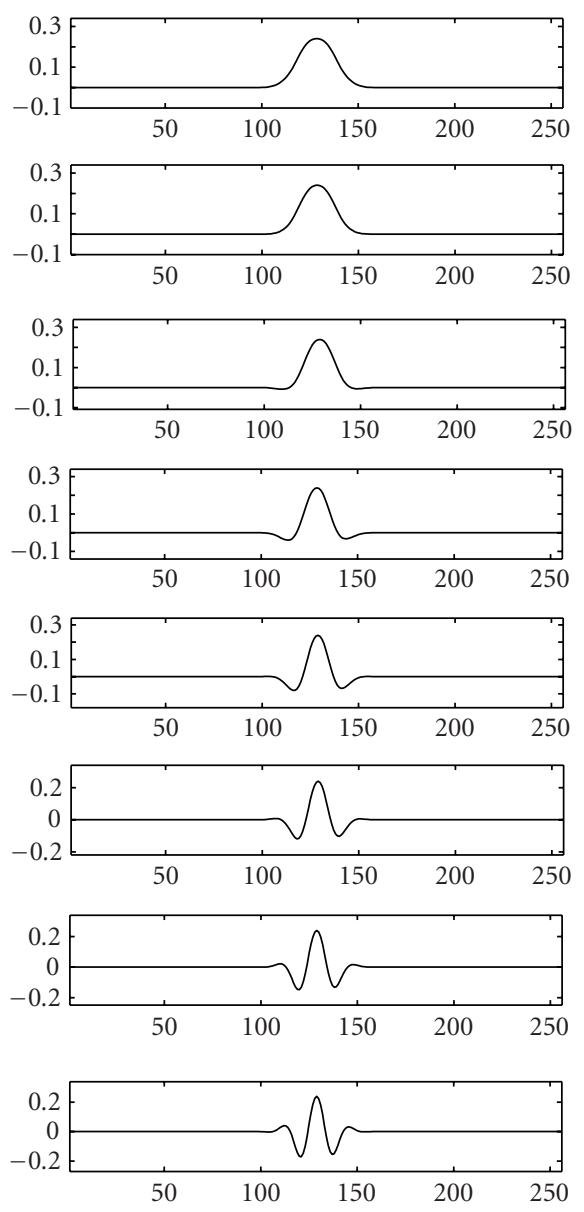

(a)
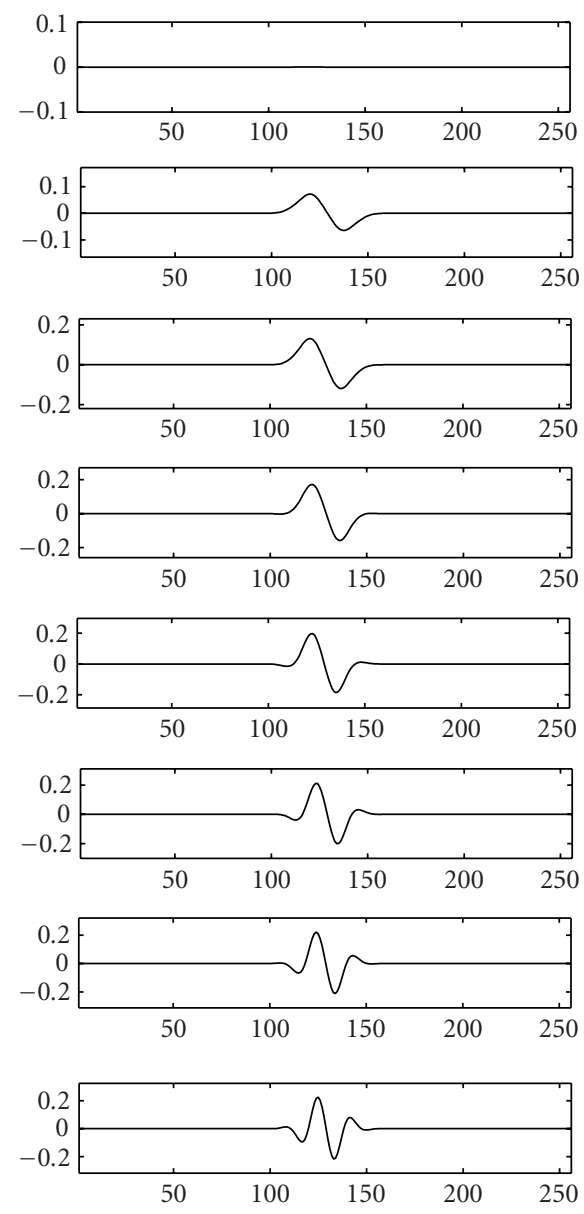

(b)

FIgURE 3: The 8 basic dual multi-Gabor windows at scale $m=1: T_{1}=16, N_{1}=128$. (a) Real part of the basic dual windows. (b) Imaginary part of the basic dual windows.

Numerical examples are provided. Proportional nonuniform MGEs eliminate unnecessary redundancies due to superfluous overlapping using identical TF shifts among all windows (in uniform MGEs). This substantially reduces the cost of post-decomposition processing, which is valuable in applications of TF signal analysis and processing. Applications of proportional nonuniform MGEs remain an open area. Practical benefits using such a more natural MGE system are expected.

\section{APPENDICES}

\section{A. PROOFS OF THEOREMS 1 AND 2}

\section{A.1. Outline of a proof of Theorem 1}

The proof is in line with similar results for continuous cases; see, for example, [23]. Again, we assume that $T_{m}$ and $N_{m}$ both divide $L$ so that $L=T_{m} K_{m}$ and $L=N_{m} P_{m}$ for all window indices $m$. We further agree that the translate index $k=k_{m}$ and the modulate index $j=j_{m}$, although for simplicity, the subindex $m$ for $k$ and $j$ or their substitutes are omitted in the presentation. Now for all $x \in \mathscr{H}_{L}$, consider

$$
\begin{aligned}
\sum_{m=0}^{M-1} & \sum_{j=0}^{N_{m}-1} \sum_{k=0}^{K_{m}-1}\left|\left\langle x, g_{j k}^{(m)}\right\rangle\right|^{2} \\
= & \sum_{m=0}^{M-1} \sum_{j=0}^{N_{m}-1} \sum_{k=0}^{K_{m}-1}\left|\sum_{t=0}^{L-1} x(t) \overline{g^{(m)}\left(t-k T_{m}\right)} e^{-\left(2 \pi i / N_{m}\right) j t}\right|^{2} \\
= & \sum_{m=0}^{M-1} \sum_{k=0}^{K_{m}-1} \sum_{t=0}^{L-1} \sum_{\tau=0}^{L-1} x(t) \overline{x(\tau)} \overline{g^{(m)}\left(t-k T_{m}\right)} g^{(m)}\left(\tau-k T_{m}\right) \\
& \times \sum_{j=0}^{N_{m}-1} e^{-\left(2 \pi i / N_{m}\right) j(t-\tau)}
\end{aligned}
$$

Notice that for each $0 \leq \tau \leq L-1$,

$$
\begin{array}{r}
\sum_{j=0}^{N_{m}-1} e^{\left(-2 \pi i / N_{m}\right) j(t-\tau)}=N_{m} \sum_{l=-\left[\tau / N_{m}\right]}^{P_{m}-\left[\tau / N_{m}\right]-1} \delta\left(t-\tau-l N_{m}\right), \\
0 \leq t \leq L-1,
\end{array}
$$


where $\delta(a-b)=1$ if $a=b, \delta(a-b)=0$ if $a \neq b$, and $[q / N]$ is the greatest integer less than or equal to $q / N$. So now, for given $\tau$,

$$
\begin{aligned}
& \sum_{m=0}^{M-1} \sum_{j=0}^{N_{m}-1} \sum_{k=0}^{K_{m}-1}\left|\left\langle x, g_{j k}^{(m)}\right\rangle\right|^{2} \\
&= \sum_{m=0}^{M-1} \sum_{k=0}^{K_{m}-1} \sum_{\tau=0}^{L-1} \sum_{l=-\left[q / N_{m}\right]}^{P_{m}-\left[q / N_{m}\right]-1} N_{m} x\left(\tau+l N_{m}\right) \overline{x(\tau)} \\
& \times \overline{g^{(m)}\left(\tau+l N_{m}-k T_{m}\right)} \\
& \times g^{(m)}\left(\tau-k T_{m}\right) \\
&= \sum_{\tau=0}^{L-1}|x(\tau)|^{2} \cdot\left(\sum_{m=0}^{M-1} N_{m} \sum_{k=0}^{K_{m}-1}\left|g^{(m)}\left(\tau-k T_{m}\right)\right|^{2}\right) \\
&+\sum_{m=0}^{M-1} N_{m} \sum_{k=0}^{K_{m}-1} \sum_{l=1}^{P_{m}-1} \sum_{\tau=0}^{L-1} \frac{x(\tau)}{x\left(\tau+l N_{m}\right)} \times\left(g^{(m)}\left(\tau-k T_{m}\right)\right. \\
&\left.\times \overline{g^{(m)}\left(\tau+l N_{m}-k T_{m}\right)}\right),
\end{aligned}
$$

where, at the last step, we have used the periodicity of sequences $x$ and $g$, and rearranged the summation over $l$ from 0 to $P_{m}-1$. is,

Now, assume that condition (1) holds in Theorem 1, that

$$
A \leq \sum_{m=0}^{M-1} N_{m} \sum_{k=0}^{K_{m}-1}\left|g^{(m)}\left(\tau-k T_{m}\right)\right|^{2} \leq B .
$$

By Cauchy-Schwartz inequality applied to the second part of (A.3), the above calculation shows that

$$
\begin{aligned}
& \sum_{m=0}^{M-1} \sum_{j k}\left|\left\langle x, g_{j k}^{(m)}\right\rangle\right|^{2}>A\|x\|^{2} \\
& -\sum_{m=0}^{M-1} N_{m} \sum_{l=1}^{P_{m}-1}\left(\max _{0 \leq \tau \leq L-1} \mid \sum_{k=0}^{K_{m}-1} g^{(m)}(\tau-k T)\right. \\
& \left.\times \frac{g^{(m)}(\tau+l N-k T)}{} \mid\right)\|x\|^{2},
\end{aligned}
$$

where the summation ranges over $j$ and $k$ are indicated in previous steps. Therefore, if condition (2) of Theorem 1 is satisfied, the lower frame bound condition is established.

\section{A.2. Outlines of the proof of Theorem 2}

The proof in Appendix A.1 up to and including (A.3) holds. Define now

$$
G_{l}^{(m)}(\tau) \equiv \sum_{k=0}^{K_{m}-1} g^{(m)}\left(\tau-k T_{m}\right) \overline{g^{(m)}\left(\tau+l N_{m}-k T_{m}\right)}
$$

and denote by $I$ the second part of (A.3). Then

$$
\begin{aligned}
I= & \sum_{m=0}^{M-1} N_{m} \sum_{l=1}^{P_{m}-1} \sum_{\tau=0}^{L-1} \overline{x(\tau)} x\left(\tau+l N_{m}\right) G_{l}^{(m)}(\tau) \\
\leq & \sum_{m=0}^{M-1} N_{m}\left(\sum_{l=1}^{P_{m}-1} \sum_{\tau=0}^{L-1}|x(\tau)|^{2}\left|G_{l}^{(m)}(\tau)\right|\right)^{1 / 2} \\
& \times\left(\sum_{l=1}^{P_{m}-1} \sum_{\tau=0}^{L-1}\left|x\left(\tau+l N_{m}\right)\right|^{2}\left|G_{l}^{(m)}(\tau)\right|\right)^{1 / 2} \\
= & \sum_{m=0}^{M-1} N_{m}\left(\sum_{l=1}^{P_{m}-1} \sum_{\tau=0}^{L-1}|x(\tau)|^{2}\left|G_{l}^{(m)}(\tau)\right|\right)^{1 / 2} \\
& \times\left(\sum_{l=1}^{P_{m}-1} \sum_{\tau=0}^{L-1}|x(\tau)|^{2}\left|G_{l}^{(m)}\left(\tau-l N_{m}\right)\right|\right)^{1 / 2} \\
= & \sum_{m=0}^{M-1} N_{m}\left(\sum_{\tau=0}^{L-1}|x(\tau)|^{2} \sum_{l=1}^{P_{m}-1}\left|G_{l}^{(m)}(\tau)\right|\right)^{1 / 2} \\
& \times\left(\sum_{\tau=0}^{L-1}|x(\tau)|^{2} \sum_{l=1}^{P_{m}-1}\left|G_{l}^{(m)}\left(\tau-l N_{m}\right)\right|\right)^{1 / 2} .
\end{aligned}
$$

Note that it is easy to verify that

$$
\sum_{l=1}^{P_{m}-1}\left|G_{l}^{(m)}\left(\tau-l N_{m}\right)\right|=\sum_{l=1}^{P_{m}-1}\left|G_{l}^{(m)}(\tau)\right| .
$$

Therefore,

$$
I \leq \sum_{m=0}^{M-1} N_{m}\left(\sum_{\tau=0}^{L-1}|x(\tau)|^{2} \sum_{l=1}^{P_{m}-1}\left|G_{l}^{(m)}(\tau)\right|\right)
$$

Hence (A.3) yields

$$
\begin{aligned}
& \sum_{m} \sum_{j k}\left|\left\langle x, g_{j k}^{(m)}\right\rangle\right|^{2} \\
& \geq \sum_{\tau=0}^{L-1}|x(\tau)|^{2} \\
& \quad \times \sum_{m=0}^{M-1} N_{m}\left(\sum_{k=0}^{K_{m}-1}\left|g^{(m)}\left(\tau-k T_{m}\right)\right|^{2}\right. \\
& \quad-\sum_{l=1}^{P_{m}-1} \mid \sum_{k=0}^{K_{m}-1} g^{(m)}\left(\tau-k T_{m}\right) \\
& \left.\times \overline{g^{(m)}\left(\tau+l N_{m}-k T_{m}\right)} \mid\right)
\end{aligned}
$$$$
\geq a\|x\|^{2},
$$

by condition (1) of Theorem 2. Similarly, condition (2) is obtained from (A.3) by adding $I$ to the first term of (A.3). 


\section{B. PROOF OF THEOREM 3}

We have assumed that $N=N_{0}$ is the smallest number of frequency bins corresponding to the window index 0 , and that $T=T_{M-1}$ is the largest time-translation parameter corresponding to the window index $M-1$. The frame operator $S$ is defined by (12). We will also be using the notation $e_{p / N} \tau_{q T} x \equiv x(\cdot-q T) e^{2 \pi i p(\cdot) / N}$.

Consider

$$
\begin{gathered}
S e_{u / N} \tau_{v T} x(t) \\
\begin{aligned}
=\sum_{m} \sum_{j k}\left\langle x(\cdot-v T) e^{2 \pi i u(\cdot) / N}, g^{(m)}\left(\cdot-k T_{m}\right) e^{2 \pi i j(\cdot) / N_{m}}\right\rangle \\
\quad \times g^{(m)}\left(t-k T_{m}\right) e^{2 \pi i j t / N_{m}} \\
=\sum_{m} \sum_{j k}\left\langle x(\cdot-v T), g^{(m)}\left(\cdot-k T_{m}\right) e^{2 \pi i\left(j-u \alpha^{m}\right)(\cdot) / N_{m}}\right\rangle \\
\quad \times g^{(m)}\left(t-k T_{m}\right) e^{2 \pi i j t / N_{m}} \\
=\sum_{m} \sum_{j k}\left\langle x, g^{(m)}\left(\cdot-\left(k-v \beta^{M-m-1}\right) T_{m}\right) e^{2 \pi i\left(j-u \alpha^{m}\right)(\cdot+v T) / N_{m}}\right\rangle \\
\quad \times g^{(m)}\left(t-k T_{m}\right) e^{2 \pi i j t / N_{m}} \\
=\sum_{m} \sum_{j k}\left\langle x, g^{(m)}\left(\cdot-\left(k-v \beta^{M-m-1}\right) T_{m}\right) e^{2 \pi i\left(j-u \alpha^{m}\right)(\cdot) / N_{m}}\right\rangle \\
\quad \times e^{-2 \pi i\left(j-u \alpha^{m}\right) v T / N_{m}} g^{(m)}\left(t-k T_{m}\right) e^{2 \pi i j t / N_{m}} \\
=\sum_{m} \sum_{j k}\left\langle x, g^{(m)}\left(\cdot-\left(k-v \beta^{M-m-1}\right) T_{m}\right) e^{2 \pi i\left(j-u \alpha^{m}\right)(\cdot) / N_{m}}\right\rangle \\
\times e_{u / N} \tau_{v T}\left[g^{(m)}\left(t-\left(k-v \beta^{M-m-1}\right) T_{m}\right) e^{-2 \pi i\left(j-u \alpha^{m}\right) t / N_{m}}\right]
\end{aligned}
\end{gathered}
$$

where we have used the fact that

$$
\begin{aligned}
e_{u / N_{m}} \tau_{v T_{m}} g^{(m)}(t) & \\
=e_{u / N} \tau_{v T}[ & g^{(m)}\left(t-\left(k-v \beta^{M-m-1}\right) T_{m}\right) \\
& \left.\times e^{-2 \pi i\left(j-u \alpha^{m}\right) t / N_{m}}\right] e^{-2 \pi i\left(j-u \alpha^{m}\right) v T / N_{m}}
\end{aligned}
$$

in canceling out an exponential factor. Hence, (B.1) continues to read

$$
\begin{aligned}
& S e_{u / N} \tau_{v T} x \\
& =e_{u / N} \tau_{v T}\left[\sum_{m} \sum_{j k}\left\langle x, e_{\left(j-u \alpha^{m}\right) / N_{m}} \tau_{\left(k-v \beta^{M-m-1}\right) T m} g^{(m)}\right\rangle\right. \\
& \left.\quad \times e_{\left(j-u \alpha^{m}\right) / N_{m}} \tau_{\left(k-v \beta^{M-m-1}\right) T_{m}} g^{(m)}\right] \\
& =e_{u / N} \tau_{v T}\left[\sum_{m} \sum_{j k}\left\langle x, e_{j / N m} \tau_{k T m} g^{(m)}\right\rangle e_{j / N m} \tau_{k T_{m}} g^{(m)}\right] \\
& =e_{u / N} \tau_{v T} S x,
\end{aligned}
$$

where in the second step, we have used the periodicity assumption of the window functions $g^{(m)}$ for each $m$.
So now if, for $0 \leq p \leq \alpha^{m}-1$ and $0 \leq q \leq \beta^{M-m-1}-1$,

$$
x=S^{-1}\left[g^{(m)}\left(\cdot-q T_{m}\right) e^{2 \pi i p(\cdot+v T) / N_{m}}\right],
$$

then

$$
\begin{aligned}
S\left(e_{u / N}\right. & \left.\tau_{v T} S^{-1}\left[g^{(m)}\left(\cdot-q T_{m}\right) e^{2 \pi i p(\cdot+v T) / N_{m}}\right]\right) \\
& =e_{u / N} \tau_{v T} S\left(S^{-1}\left[g^{(m)}\left(\cdot-q T_{m}\right) e^{2 \pi i p(\cdot+v T) / N_{m}}\right]\right) \\
& =e_{u / N} \tau_{v T}\left[g^{(m)}\left(\cdot-q T_{m}\right) e^{2 \pi i p(\cdot+v T) / N_{m}}\right] \\
& =e_{\left(u \alpha^{m}+p\right) / N_{m}} \tau_{\left(\nu \beta^{M-m-1}+q\right) T_{m}} g^{(m)} .
\end{aligned}
$$

Therefore, writing $j=u \alpha^{m}+p$ and $k=v \beta^{M-m-1}+q$, where $u=0,1, \ldots, N-1, v=0,1, \ldots, L / T-1, p=0,1, \ldots, \alpha^{m}-1$, and $q=0,1, \ldots, \beta^{M-m-1}-1$, we see that $0 \leq j \leq N_{m}-1$, and $0 \leq k \leq L / T_{m}-1$ for all $m$, and

$$
\begin{aligned}
S^{-1}( & \left.e_{j / N_{m}} \tau_{k T_{m}} g^{(m)}\right) \\
& =S^{-1}\left(e_{\left(u \alpha^{m}+p\right) / N_{m}} \tau_{\left(v \beta^{M-m-1}+q\right) T_{m}} g^{(m)}\right) \\
& =e_{u / N} \tau_{v T} S^{-1}\left[g^{(m)}\left(\cdot-q T_{m}\right) e^{2 \pi i p(\cdot+v T) / N_{m}}\right] \\
& =e_{u / N} \tau_{v T}\left[e^{2 \pi i p v T / N_{m}} S^{-1}\left(g^{(m)}\left(\cdot-q T_{m}\right) e^{2 \pi i p(\cdot) / N_{m}}\right)\right] .
\end{aligned}
$$

This implies that the dual-frame sequence

$$
\left\{S^{-1}\left(e_{j / N_{m}} \tau_{k T_{m}} g^{(m)}\right): m, j, k\right\}
$$

is formed by translations by $T$ and complex modulations by the frequency parameter $N$ of a few basic dual window functions $\left\{S^{-1}\left[g^{(m)}\left(\cdot-q T_{m}\right) e^{2 \pi i p(\cdot) / N_{m}}\right]: m, p, q\right\}$ with a multiple constant. The theorem is thereby established.

\section{OUTLINE OF THE PROOF OF COROLLARY 1}

Consider, similarly,

$$
\begin{aligned}
& S \tau_{q T_{0}} x(t) \\
&=\sum_{m} \sum_{j k}\left\langle x\left(\cdot-q T_{0}\right), g^{(m)}\left(\cdot-k T_{m}\right) e^{2 \pi i j(\cdot) / N_{m}}\right\rangle \\
& \times g^{(m)}\left(t-k T_{m}\right) e^{2 \pi i j t / N_{m}} \\
&=\sum_{m} \sum_{j k}\left\langle x, g^{(m)}\left(\cdot+q T_{0}-k T_{m}\right) e^{2 \pi i j\left(\cdot+q T_{0}\right) / N_{m}}\right\rangle \\
& \times g^{(m)}\left(t-k T_{m}\right) e^{2 \pi i j t / N_{m}} \\
&=\sum_{m} \sum_{j k}\left\langle x, g^{(m)}\left(\cdot+q T_{0}-k T_{m}\right) e^{2 \pi i j(\cdot) / N_{m}}\right\rangle \\
& \times e^{-2 \pi i j q T_{0} / N_{m}} g^{(m)}\left(t-k T_{m}\right) e^{2 \pi i j t / N_{m}} \\
&=\tau_{q T_{0}}\left(\sum_{m} \sum_{j k}\left\langle x, g^{(m)}\left(\cdot+q T_{0}-k T_{m}\right) e^{2 \pi i j(\cdot) / N_{m}}\right\rangle\right. \\
&\left.\times g^{(m)}\left(t+q T_{0}-k T_{m}\right) e^{2 \pi i j t / N_{m}}\right)
\end{aligned}
$$$$
=\tau_{q T_{0}}\left(S_{q T_{0}} x\right) .
$$ 
So now, for $x=S_{q T_{0}}^{-1} g^{(0)}$,

$$
S \tau_{q T_{0}}\left(S_{q T_{0}}^{-1} g^{(0)}\right)=\tau_{q T_{0}} g^{(0)}
$$

or

$$
\tilde{\gamma}^{(0,0, q)}=S^{-1}\left(\tau_{q T_{0}} g^{(0)}\right)=\tau_{q T_{0}}\left(S_{q T_{0}}^{-1} g^{(0)}\right) .
$$

\section{ACKNOWLEDGMENT}

Shidong Li is partially supported by NSF Grant DMS0103762, USA.

\section{REFERENCES}

[1] M. J. Bastiaans, "Gabor's expansion of a signal into Gaussian elementary signals," Proceedings of the IEEE, vol. 68, no. 4, pp. 538-539, 1980.

[2] J. J. Benedetto, "Gabor representations and wavelets," Contemporary Mathematics, vol. 19, pp. 9-27, 1989.

[3] J. J. Benedetto, C. Heil, and D. F. Walnut, "Gabor systems and the Balian-Low theorem," in Gabor Analysis and Algorithms: Theory and Applications, H. G. Feichtinger and T. Strohmer, Eds., pp. 85-122, Birkhäuser, Boston, Mass, USA, 1997.

[4] J. J. Benedetto, C. Heil, and D. F. Walnut, "Differentiation and the Balian-Low theorem," Journal of Fourier Analysis and Applications, vol. 1, no. 4, pp. 355-402, 1995.

[5] I. Daubechies, H. Landau, and Z. Landau, "Gabor timefrequency lattices and the Wexler-Raz identity," Journal of Fourier Analysis and Applications, vol. 1, no. 4, pp. 437-478, 1995.

[6] H. G. Feichtinger and K. Grochenig, "Gabor wavelets and the Heisenberg group: Gabor expansions and short time Fourier transform from the group theoretical point of view," in Wavelets-A Tutorial in Theory and Applications, C. K. Chui, Ed., vol. 2, pp. 359-397, Academic Press, Boston, Mass, USA, 1992.

[7] T. Ebrahimi and M. Kunt, "Image compression by Gabor expansion,” Optical Engineering, vol. 30, no. 7, pp. 873-880, 1991.

[8] A. J. E. M. Janssen, "Gabor representation of generalized functions," Journal of Mathematical Analysis and Applications, vol. 83, no. 2, pp. 377-394, 1981.

[9] C. Heil and D. Walnut, "Gabor and wavelet expansions," in Recent Advances in Fourier Analysis and Its Applications, J. S. Byrnes and J. L. Byrnes, Eds., vol. 315 of NATO Adv. Sci. Inst. Ser. C: Math. Phys. Sci., pp. 441-454, Kluwer Academic, Dordrecht, the Netherlands, 1990.

[10] A. J. E. M. Janssen, "Duality and biorthogonality for WeylHeisenberg frames," Journal of Fourier Analysis and Applications, vol. 1, no. 4, pp. 403-436, 1995.

[11] S. Li, "Fast and parametric algorithm for discrete Gabor expansions and the role of various dual windows," in Wavelet Applications II, H. H. Szu, Ed., vol. 2491 of Proceedings of SPIE, pp. 935-946, Orlando, Fla, USA, April 1995.

[12] S. Li and D. M. Healy Jr., "A parametric class of discrete Gabor expansions," IEEE Trans. Signal Processing, vol. 44, no. 2, pp. 201-211, 1996.

[13] S. Qian, K. Chen, and S. Li, "Optimal biorthogonal sequence for finite discrete-time Gabor expansion," Signal Processing, vol. 27, no. 2, pp. 177-185, 1992.
[14] R. Balan, I. Daubechies, and V. Vaishampayan, "The analysis and design of windowed fourier frame based multiple description source coding schemes," IEEE Transactions on Information Theory, vol. 46, no. 7, pp. 2491-2536, 2000.

[15] R. Balan, P. Casazza, C. Heil, and Z. Landau, "Excesses of Gabor frames," Applied \& Computational Harmonic Analysis, vol. 14, no. 2, pp. 87-106, 2003.

[16] J. Wexler and S. Raz, "Discrete Gabor expansions," Signal Processing, vol. 21, no. 3, pp. 207-220, 1990.

[17] H. G. Feichtinger and T. Strohmer, Eds., Gabor Analysis and Algorithms: Theory and Applications, Birkhäuser, Boston, Mass, USA, 1997.

[18] S. Li, "Discrete multi-Gabor expansions," IEEE Transactions on Information Theory, vol. 45, no. 6, pp. 1954-1967, 1999.

[19] Y. Y. Zeevi, M. Zibulski, and M. Porat, "Multi-window Gabor schemes in signal and image representations," in Gabor Analysis and Algorithms: Theory and Applications, H. G. Feichtinger and T. Strohmer, Eds., pp. 381-407, Birkhäuser, Boston, Mass, USA, 1997.

[20] M. Zibulski and Y. Y. Zeevi, "Analysis of multiwindow Gabortype schemes by frame methods," Applied \& Computational Harmonic Analysis, vol. 4, no. 2, pp. 188-221, 1997.

[21] S. Li, "On general frame decompositions," Numerical Functional Analysis and Optimization, vol. 16, no. 9-10, pp. 11811191, 1995.

[22] P. G. Casazza and O. Christensen, "Weyl-Heisenberg frames for subspaces of $L^{2}(\mathbf{R})$," Proc. Amer. Math. Soc., vol. 129, no. 1, pp. 145-154, 2001.

[23] C. Heil and D. Walnut, "Continuous and discrete wavelet transforms," SIAM Review, vol. 31, no. 4, pp. 628-666, 1989.

Shidong Li received the B.S. degree in electrical engineering from the Hefei University of Technology, China, in 1982, and the M.S. degree in electrical engineering from the Graduate School of the Chinese Academy of Sciences in 1985. He received his second M.S. degree in applied mathematics in 1989, and the Ph.D. degree in applied mathematics in 1993 from the Graduate School, University of Maryland, Baltimore. From 1985

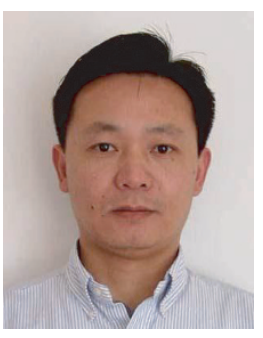
to 1987, he was a Lecturer at the Graduate School of the Chinese Academy of Sciences. He was a Visiting Assistant Professor at Dartmouth College from 1993 till 1994 and the University of Maryland, College Park, from 1994 till 1996. He has been with San Francisco State University since 1996. Dr. Li is currently an Associate Professor of mathematics. His major research interests include wavelet theories, frame and frame extensions and their applications in signal analysis and processing, time-frequency representations, Gabor expansions, signal processing in digital communication systems, and applied mathematics. 